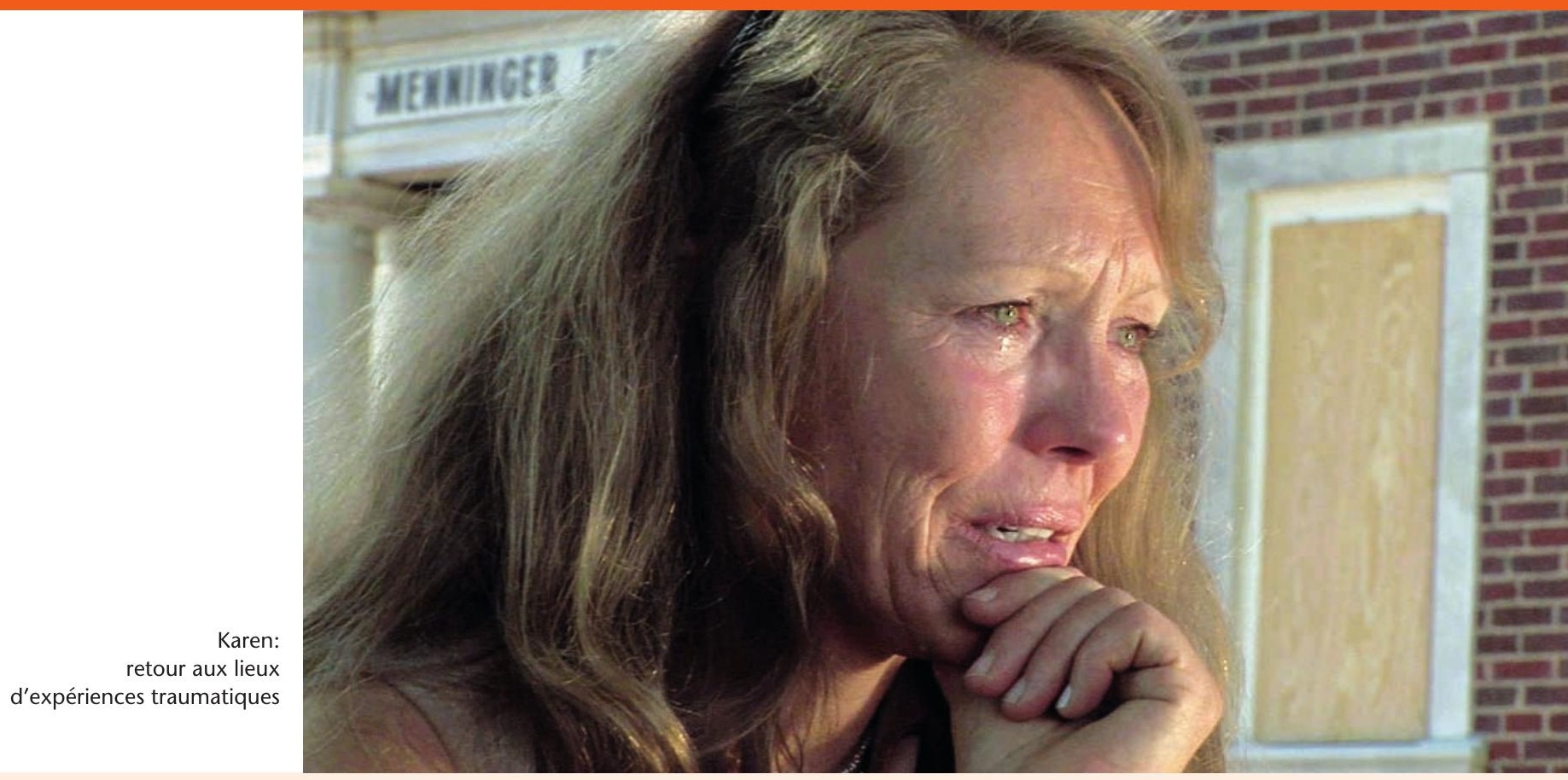

\title{
Someone beside you - Une approche filmée de la psychose
}

Bruno Kesseli
Une femme est à la fenêtre. Un événement quotidien, en principe non spectaculaire, qu'Edgar Hagen, dans son film «Someone beside you», présente comme un moment dont la tension est palpable. La femme et la fenêtre ont ensemble une histoire: Karen est revenue, après plus de trois décennies, à l'endroit où, jeune fille, elle tenta de se suicider. Désespérée, déchirée, «psychotique», elle ouvrit alors la fenêtre au $10^{\mathrm{e} e \text { étage }}$ d'un hôtel américain à Topeka et se jeta dans le vide.

Le film de Hagen contient toute une série de séquences qui absorbent entièrement le spectateur, parce que la distance par rapport à l'événement sur l'écran disparaît soudainement et que l'on a l'impression de vivre de l'intérieur les sentiments des protagonistes, et peut-être même des éléments de leurs pensées. Au début du film, la discussion animée entre le psychiatre Jakob Litschig et Kaspar - tous deux ayant une expérience notable de la psychose - en est un autre exemple: l'intensité de cet épisode n'est pas facile à supporter, notamment de par le fait qu'il recèle un potentiel de violence difficilement mesurable. D'autres scènes sont tout aussi bouleversantes, dans un autre registre, comme celle où Andrea parle de sa vie psychotique. Pendant ses phases de maladie, elle était persuadée de vivre dans un monde plein de cadavres et à la clinique, elle avait le sentiment que les repas étaient faits de morceaux de corps morts. Qu'est-ce qu'une psychose? Sous quelles conditions apparaît-elle? Que se passe-t-il avec des personnes qui dérapent, débloquent, perdent l'entendement? Dans son film, Hagen aborde ces questions avec tact. Au contraire du metteur en scène Ron Howard, qui dans son film «A Beautiful Mind», fait acte de virtuosité, mais qui, avec force concessions aux «goûts du public», fait du cinéma hollywoodien, Hagen aborde la psychose, thème du film, de manière beaucoup plus réservée. Il ne tente pas de présenter le monde intérieur de ses protagonistes sur le plan de l'imagination fictionnelle ou sous une forme dramatique. Mais précisément parce qu'il reste en «dehors», qu'il ne pousse pas les portraits et ne veut pas imposer au public des images et des sons «intérieurs», il crée un espace où ce monde peut s'épanouir et être vécu par le spectateur.

L'auteur est en route avec ses personnages principaux, il cherche parfois avec eux des endroits qui ont pour eux une signification essentielle par rapport à leur vécu psychotique. La confiance qu'ils ont en lui leur permet de se com- 
porter naturellement et de parler de manière bouleversante de leur vie et de leurs expériences de la crise psychotique. La force de l'image est conjuguée à la force des mots de façon magistrale: dans les visages et les gestes des personnages qui ont vécu une psychose, nous trouvons l'authentification de leurs paroles. Leurs personnalités deviennent visibles, et l'on comprend pourquoi il leur est très difficile de répondre aux exigences que la société moderne pose à des individus «fonctionnant» normalement.

Bien que le thème soit porteur de gravité, la manière dont Hagen l'aborde est fondamentalement optimiste. Il est philosophe et en tant que tel, il s'intéresse aux conditions de l'existence humaine et à l'esprit de l'homme. La psychose peut à l'extrême être comprise comme s'insérant dans la continuité des états d'esprit qui font partie du «bagage de base» de l'être humain. Cette réflexion est sous-jacente au film et devient plus ou moins explicite à travers les personnages. Le fait que Hagen ait trouvés ces derniers en premier lieu dans le projet Windhorse à Boulder, Colorado, n'est sûrement pas un hasard, mais relève plutôt d'une sorte de phénomène de résonance.

Edward Podvoll, psychiatre, psychanalyste et lama bouddhiste, fondateur du projet Windhorse, concevait les crises psychotiques comme des crises spirituelles, dans lesquelles le noyau sain de tout être humain est simplement plus difficile à trouver. La tâche thérapeutique centrale était, selon lui, de parvenir à ce noyau et de le renforcer. Les concepts de Podvoll sont fortement imprégnés du bouddhisme tibétain qu'il ne considère pas comme une religion, mais comme une tradition de 2500 ans de recherche sur l'esprit humain. La rencontre de Hagen filmant Podvoll a eu lieu quelques semaines avant sa mort. Le flegme tranquille que ce dernier montra alors qu'il souffrait d'un cancer donne à ses répliques une force saisissante.

Hagen suit une voie logique dans son film, aussi bien quant à la teneur qu'à la forme. Il a le courage d'explorer à fond les questions essentielles. Et le courage aussi de ne pas vouloir traiter exhaustivement le sujet de la psychose. La biochimie de la psychose, les débats psychopathologiques, les classifications, la mise en parallèle et l'évaluation de concepts thérapeutiques, la critique de la psychiatrie sont des thèmes qui n'interviennent pas dans «Someone beside you». Heureusement, pourrait-on dire. Car, du moins peut-on le supposer, ce n'est qu'en se restreignant de la sorte que l'on pouvait atteindre la profondeur et l'équilibre qui caractérisent ce film.

«Someone beside you» est visible dans plusieurs villes de Suisse alémanique à partir du 29 mars. La programmation du film en Suisse romande n'est pas encore fixée.

Dans plusieurs villes suisses alémaniques, des tables rondes et des projections spéciales de «Someone beside you» sont annoncées sur internet à l'adresse suivante: www.someonebesideyou.com.

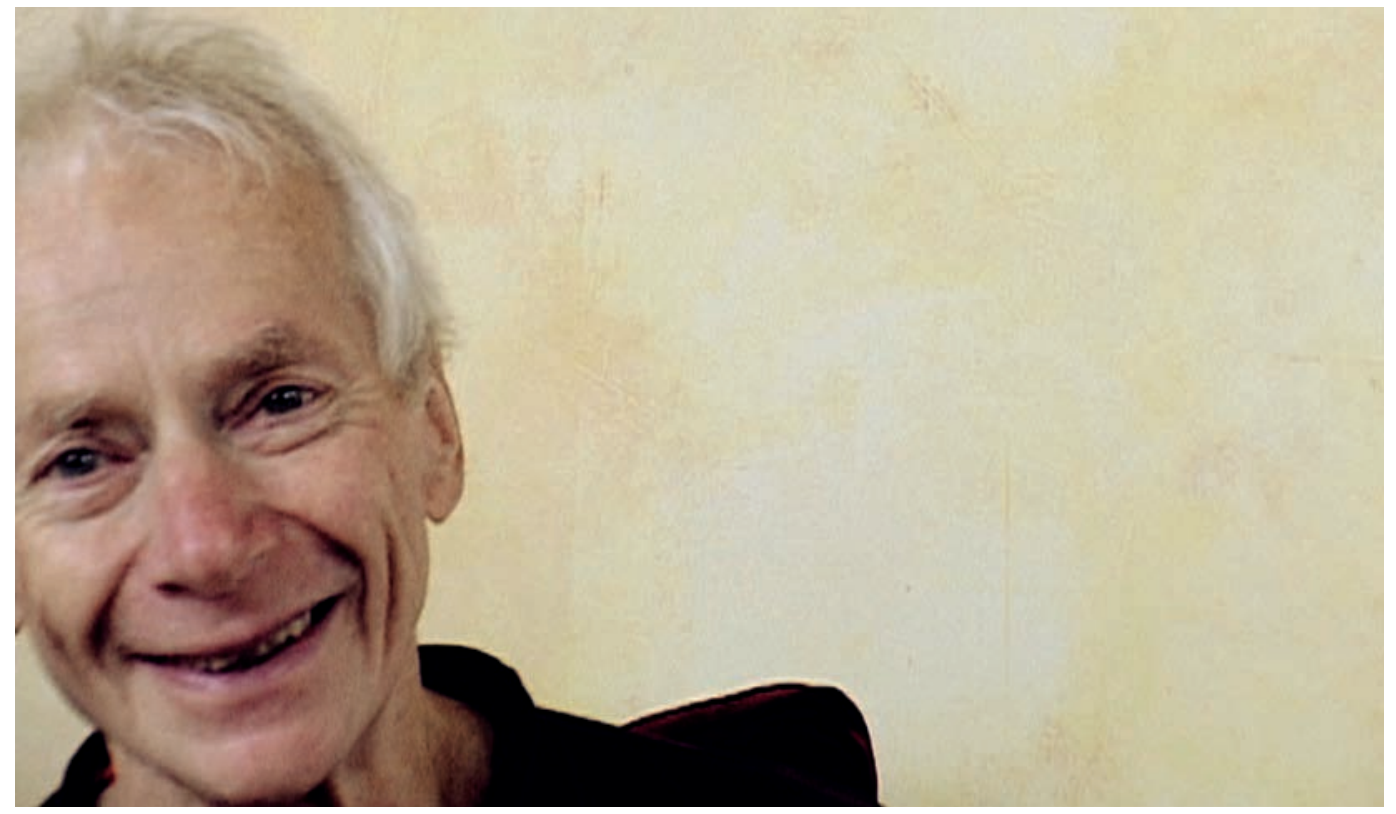

Edward Podvoll, fondateur du projet Windhorse 